\title{
A Comparative Review of the Effect of Microcystin-LR on the Proteome
}

\author{
Richard D. Welten ${ }^{1}$ (D) Julie P. Meneely ${ }^{1} \cdot$ Christopher T. Elliott $^{1}$
}

Received: 18 September 2018 / Revised: 1 February 2019 / Accepted: 7 February 2019 / Published online: 18 February 2019

(c) The Author(s) 2019

\begin{abstract}
Cyanobacterial toxins are a growing threat to human and animal welfare in many parts of the world. Microcystin-LR is the most widely studied of the cyanotoxins and has been implicated with hepatotoxicity, neuropathology, and genotoxicity. Numerous studies investigated the effect of microcystin-LR exposure on the proteome using various animal models, and together they form a large database of potential protein biomarkers. However, it is extremely difficult to establish which proteins are specifically affected by microcystin-LR, and which represent a more general toxin response. The goal of this review was to filter out inconsistently reported protein abundancy changes after microcystin-LR exposure. We explored online search engines for studies investigating the effect of microcystin-LR toxicity on the proteome. The selected studies were examined to find overlapping protein abundancy changes. The protein names, their synonyms, and relevant orthologues were used as search terms. This review has produced, for the first time, a comprehensive list of proteins whose abundancies changed in at least two proteomic studies investigating microcystin-LR toxicity in rodents and zebrafish. Proteins involved in oxidoreductase activity and cytoskeletal processes are persistently affected by microcystin-LR exposure. Several oxidative stress markers are consistently altered across multiple proteomic studies, which correlates with findings from epidemiological studies that linked chronic microcystin exposure to increased incidences of liver and colorectal cancer. This study unveils which proteins' abundancies are consistently altered after microcystin-LR exposure and opens new doors to understanding the mechanisms behind microcystin-LR toxicity.
\end{abstract}

Keywords microcystin-LR $\cdot$ microcystins $\cdot$ cyanotoxins $\cdot$ cyanobacteria $\cdot$ proteomics $\cdot$ proteome

\section{Introduction}

The growing worldwide presence of toxin-producing cyanobacteria has become an increasing concern. Also known as blue-green algae, these photosynthetic prokaryotes can be found in lakes, ponds, and rivers across the globe (van Apeldoorn et al. 2007). They form a serious threat to the environment and human health when undergoing proliferation, a process called 'blooming'. Increased eutrophication of freshwater bodies, often caused by industrial pollution, may lead to cyanobacterial blooming (Pitois et al. 2001). Fueled by an abundance of nutrients, cyanobacteria not only increase in numbers exponentially, certain species additionally produce an increasing amount of toxic secondary metabolites. These

Richard D. Welten

rwelten01@qub.ac.uk

1 Institute for Global Food Security, School of Biological Sciences, Queen's University Belfast, Belfast BT7 1NN, Northern Ireland, UK compounds are also known as cyanotoxins (van Apeldoorn et al. 2007).

Microcystins are among the most studied cyanotoxins. With a molecular mass of around $1000 \mathrm{Da}$, microcystins are relatively large natural products. They contain seven peptide-linked amino acids, of which the terminal amino acids are condensed, making microcystin a cyclic compound (Meneely and Elliott 2013).

Microcystin-LR (MC-LR) is believed to be one of the most toxic variants of the microcystins. It binds to the catalytic center of serine/threonine protein phosphatases, including protein phosphatases 1 and 2A (PP1 and PP2A) (Runnegar et al. 1995). The catalytic subunits of PP1 and PP2A associate to different regulatory subunits to form holoenzymes, allowing the regulation of DNA replication, cell differentiation, metabolism, apoptosis, and other key cellular functions (Garcia et al. 2003). Because microcystins block the active sites of PP1 and PP2A, protein phosphatase activity is inhibited. This leads to hyperphosphorylation of proteins, which perturbs the phosphorylation/dephosphorylation 
balance, affecting various cellular functions PP1 and PP2A are involved in (Gehringer 2004).

\section{MC-LR Toxicity in Animals}

Multiple animal toxicology studies found dose-dependent hepatic injury and altered changes in liver enzyme levels after oral MC-LR exposure in mammals (Heinze 1999; Fawell et al. 1999; Sedan et al. 2015), though not every study reported an effect (Ueno et al. 1999). In a short-term exposure study in mice, animals were treated with MC-LR by oral gavage for 13 weeks daily (Fawell et al. 1999). Liver pathology was visible in some of the rodents $(200 \mu \mathrm{g} / \mathrm{kg}$ bodyweight concentration), though at the highest concentration $(1000 \mu \mathrm{g} / \mathrm{kg}$ bodyweight) all mice showed signs of hepatotoxicity. The no observed adverse effect level (NOAEL) of MC-LR for liver damage was $40 \mu \mathrm{g} / \mathrm{kg}$ bodyweight. Based on this NOAEL, the World Health Organization (WHO) set a guideline value for MC-LR. They applied an uncertainty factor of 1000 to the NOAEL (100 for intra- and interspecies variation, 10 for limitations in the database) and set the tolerable daily intake (TDI) at $0.04 \mu \mathrm{g} / \mathrm{kg}$ bodyweight per day (Chorus and Bartram 1999). The guideline value for MC-LR is $1 \mu \mathrm{g} / \mathrm{L}$ (cell-bound and extracellular) in drinking water (Chorus and Bartram 1999).

\section{MC-LR Toxicity in Humans}

There are various routes through which humans can be exposed to microcystins. The most direct route is through the ingestion of contaminated water, for example, by consuming compromised drinking water or by performing recreational activities in polluted waterbodies. A more indirect way of ingesting microcystins is through the consumption of microcystin-containing foods. Indeed, microcystins bioaccumulate in various animals used for human consumption, including fish (Mohamed et al. 2003; Magalhães et al. 2003; Xie et al. 2005), snails (Lance et al. 2010), and crustaceans (Magalhães et al. 2003). Microcystins also enter the human food chain when crops are irrigated using contaminated water. The toxins have been found in lettuce (Codd et al. 1999), but also in cabbage, radish, rocket, dill, and parsley (Mohamed and Al Shehri 2009).

Well-documented cases of acute microcystin toxicity in humans are scarce. However, one tragic incident in Brazil has shown the fatal effects microcystins can have on human health. In February 1996, hemodialysis patients received water that had not been properly treated (Jochimsen et al. 1998). As a result, the dialysate contained microcystins and patients reported symptoms. These included muscle weakness, visual disturbance, nausea, and vomiting (Jochimsen et al. 1998). During this outbreak, $85 \%$ of the victims experienced acute liver injury, and nearly $48 \%$ died (Pouria et al.
1998). In the month following the outbreak, patients had a more than sevenfold increase in liver function enzyme serum aspartate aminotransferase concentrations, in addition to a more than fourfold increase in total and conjugated bilirubin (Jochimsen et al. 1998). Liver histology showed acute toxic hepatitis, similar to that seen in animals exposed to microcystins (Pouria et al. 1998). Using immunoassays, it was found that microcystins were present in serum samples at concentrations ranging from below $1 \mathrm{ng} / \mathrm{mL}$ to $10 \mathrm{ng} /$ $\mathrm{mL}$, whereas in liver this was below $0.1 \mathrm{ng} / \mathrm{mg}$ to $0.4 \mathrm{ng} /$ mg liver tissue (Carmichael et al. 1996; Pouria et al. 1998). Mass spectrometry analyses confirmed the presence of microcystin-AR, MC-LR and microcystin-YR (Carmichael et al. 1996; Pouria et al. 1998).

While the fatal incident in Brazil exposed the dangers of microcystins to humans, chronic exposure to low microcystin concentrations is much more common. Several epidemiological studies have looked at the effect of oral microcystin exposure in humans. In 1983, it was reported that a drinking water supply was contaminated with water from a reservoir containing a toxic Microcystis bloom in Armidale, Australia. A retrospective study statistically analyzed the activity of liver function enzymes in exposed and non-exposed residents (Falconer et al. 1983; Falconer 2005). The authors discovered a significant increase in gamma glutamyl transferase activity in plasma samples from the exposed group during the bloom, whereas no significant alterations in activity were found 6 weeks before and six weeks after the bloom (Falconer et al. 1983). In addition, Chen and colleagues discovered irregular liver function enzyme levels in serum samples from local fishermen at Lake Chaohu, a waterbody regularly plagued by dense cyanobacterial blooms (Chen et al. 2009). The results showed a positive correlation between microcystin serum concentrations and liver function enzymes alanine aminotransferase (ALT), aspartate aminotransferase, lactate dehydrogenase, and alkaline phosphatase. In addition, Li et al. detected that children who consumed drinking water sourced from microcystin-contaminated lakes had significantly higher levels of liver function enzymes than the control population ( $\mathrm{Li}$ et al. 2011b).

These epidemiological studies revealed a correlation between microcystin exposure in humans and hepatotoxicity, further suggesting that microcystins may form a health hazard. This makes it crucial to get a better understanding of the mechanisms behind MC-LR toxicity, as well as to identify which components of the cellular machinery are affected.

\section{MC-LR and the Proteome}

There are various tools in place to study components of whole biological systems. Indeed, advancements in 'omics' technologies have enabled the analysis of a large number of molecules across different levels of biological organization. 
In toxicology, such studies have allowed the identification and quantification of biomarkers in response to xenobiotics.

A proteome study is intended to interrogate the entire set of proteins present in the model of interest (e.g., a cell, tissue or organism). In the case of toxicology studies in animals, the proteomes of a treatment group are usually compared with that of a control (untreated) group. Differences in protein levels between the treatment and control group, in theory, are caused by the administered xenobiotic. Further downstream applications are performed for protein identification and validation. Once a list of affected proteins has been made, functional analysis may reveal interactions and pathways relevant to understanding the toxin's mode of action. Thus, proteomic studies allow the investigation of a large set of proteins in response to a xenobiotic compound, making it a very useful tool for biomarker discovery.

The effect of MC-LR exposure on the proteome has been investigated in rodents and zebrafish (Table 1). While these studies give valuable insights, it is difficult to translate the results into a human scenario because of the experimental designs applied. Indeed, most studies administered MC-LR via intraperitoneal injection (IP) and used high toxin concentrations. This despite the fact that the most common route of MC-LR uptake for humans is through chronic low-dose oral exposure, e.g., by drinking from a contaminated water source. This makes it challenging to assess whether the observed effects in these studies are (1) truly representative for MC-LR, or rather caused by a non-specific toxin response due to the intense dosing nature, and (2) whether chronic low-dose oral exposure would generate similar results. Only one published proteomic study has looked at the effect of chronic oral MC-LR exposure in mammals (He et al. 2017), and the lowest concentration used was $40 \mu \mathrm{g} /$ $\mathrm{kg}$ bodyweight per day. This equates to a thousand times higher dose than the TDI of $0.04 \mu \mathrm{g} / \mathrm{kg}$ bodyweight set by the WHO, and it is questionable whether any humans or indeed animals are exposed to such high MC-LR concentrations on a frequent basis.

Therefore, the goal of this review is to provide a clearer picture of MC-LR toxicity by identifying re-occurring changes in protein abundancies reported in proteomic studies that investigated the effect of MC-LR toxicity in rodents and zebrafish, as well as to discuss how these results may relate to human exposure conditions.

\section{Methods}

Due to the high number of reported MC-LR biomarkers in the proteome, and the often extreme parameters used in the experimental designs, it is difficult to determine which effects are specific to MC-LR toxicity. We reasoned that if changes in a certain protein's abundancy were to be specific to MC-LR exposure, this trend would repeat itself over multiple studies. To this end, a table was constructed that lists proteins whose abundancies were altered in at least two proteomic studies investigating MC-LR exposure in rodents and zebrafish (Table 1). While this method does not guarantee that the proteins listed in Table 1 are specific to MC-LR toxicity, the fact that their levels were altered across multiple studies increases the likelihood. Importantly, it should be kept in mind that some of these proteins may simply represent a general toxin response, as it is hard to distinguish unique MC-LR biomarkers from proteins involved in universal toxin responses.

\section{Database Searches}

To identify relevant studies that investigated the effect of MC-LR on the proteome, search engines PubMed (https:// www.ncbi.nlm.nih.gov/pubmed/), Google Scholar (https:// scholar.google.com/), and Web of Science (https://webof knowledge.com/) were used. The latest search performed was in June 2018. The following keywords served as search terms: microcystin-LR, MC-LR, microcystin, microcystins, proteome, proteomics, systems biology, systems toxicology. Studies were considered relevant if the authors investigated the effect of MC-LR on the proteome using untargeted proteomic methods, such as 2-dimensional electrophoresis (2DE) and isobaric tags for relative and absolute quantification (iTRAQ). Only investigations that had rodents and zebrafish as the experimental model were included. Where applicable, reported accession numbers were validated on the National Center for Biotechnology Information Protein database (https://www.ncbi.nlm.nih.gov/protein/) and UniProt (https://www.uniprot.org/). Searches for individual accession numbers and protein names were performed on UniProt and Ensembl (https://www.ensembl.org/index.html, release 89) databases to find potential synonyms and orthologues. The protein names, accession numbers, synonyms, and orthologues (including their potential synonyms) were subsequently used as search terms in Acrobat Reader DC software (Adobe, version 2017.009.20044) in order to find overlapping reports of individual biomarkers among the proteomic studies. In cases where the original file was not accessible by Acrobat Reader DC software, for example, when the list of biomarkers was published in a supplementary Microsoft Word document, the file was converted to the. pdf file format that could be included to the simultaneous search. This resulted in a list of proteins whose abundancies were altered in at least two proteomic investigations studying MC-LR toxicity (Table 1 ). The protein names listed in Table 1 are those recommended by UniProt (release 2018_01) and may be different from the synonyms reported by the investigated proteomic studies. 


\section{Protein Classification}

In order to get a broader overview of the molecular functions influenced by MC-LR, classification system PANTHER (https://pantherdb.org) was used for functional classification. To this end, human orthologues of the proteins listed in Table 1 were compared to the Homo sapiens reference database (PANTHER 13.1 release). This was done separately for proteins found in the liver and brain. The resulting data were visualized (Fig. 1) using the ggplot2 package (https:// ggplot2.org) in R (https://www.r-project.org/). PANTHER's statistical overrepresentation test utility determined which molecular functions were overrepresented in the liver and brain compared to the Homo sapiens reference database (PANTHER 13.1 release). The statistical method applied by the PANTHER algorithm was the Fisher's exact test with false discovery rate (FDR) multiple test correction.

\section{Commonly Reported Protein Changes in 2DE Analyses}

In order to make a better distinction between potential MC-LR biomarkers and proteins whose abundancies change frequently across 2DE studies, the results from two studies were used (Petrak et al. 2008; Wang et al. 2009). These investigations established two lists of frequently reported protein abundancy changes regardless of experimental setup when using 2DE. Table 1 indicates which proteins overlap with those found in these studies, and are thus more likely to represent a non-specific response.

\section{Results}

Table 1 lists proteins whose abundancies changed in at least two proteomic studies investigating MC-LR toxicity in rodents or zebrafish. The data were derived from eleven studies that reported at least one overlapping protein whose abundancy changed. Five investigations applied MC-LR intraperitoneally, four zebrafish studies supplied the toxin by continuous exposure through water, one used continuous subcutaneous injection, and one administered MC-LR by oral gavage. Most studies explored the proteome using 2DE, although ITRAQ was applied as well. Organs interrogated include the liver, brain, and to a lesser extent the spleen. In the case of zebrafish, whole embryos were analyzed as well.

\section{Functional Annotation}

Using the Panther database (https://pantherdb.org/, release 13.1), functional annotation was applied to the resulting protein list to get a better overview of the molecular functions affected by MC-LR. To simplify this process, human orthologues of the found proteins were used, as well as the human reference database. The stacked bar plot in Fig. 1 illustrates the gene ontology molecular functions represented in the liver and brain. In liver, proteins affected by MC-LR were mainly involved in catalytic activity (52.5\%), binding $(25.0 \%)$, and antioxidant activity (10.0\%). Interestingly, in the brain, there was an increase in structural molecule activity when compared to liver (19.0\% in brain, $5.0 \%$ in liver), whereas other overlapping categories remained largely the same.

\section{Overrepresentation Tests}

Statistical overrepresentation tests were carried out for the molecular functions represented in liver (Table 2) and brain (Table 3). In these tests, the input list (i.e., altered liver or brain proteins from Table 1) is categorized on the basis of specific classes (e.g., molecular function). The resulting list is then compared to a reference genome, which has also been grouped based on specific classes, in order to determine which classes are under-or overrepresented in the input list (Mi et al. 2013). For liver, proteins involved in molecular functions peroxidase, antioxidant, and oxidoreductase activity were significantly overrepresented (Table 2). Catalytic activity was overrepresented as well. Molecular functions significantly overrepresented in the brain were antioxidant activity, structural constituent of the cytoskeleton, structural molecule activity, oxidoreductase activity, and catalytic activity (Table 3 ).

\section{Discussion}

This review has identified 39 proteins whose abundancies were changed in at least two proteomic studies investigating MC-LR toxicity (Table 1). Many of these studies chose experimental designs that allow high bioavailability of the toxin. Indeed, the most common administration route was by IP and the lowest toxin concentration reported for this method was $1 \mu \mathrm{g} / \mathrm{kg}$ bodyweight, which is 25 times higher than the $0.04 \mu \mathrm{g} / \mathrm{kg}$ bodyweight TDI set by the WHO. While to our knowledge MC-LR bioavailability has not been compared between animals dosed intraperitoneally and orally, it seems reasonable to assume that dosing intraperitoneally would lead to a much higher absorption rate of the toxin. The lowest dose orally administered was $40 \mu \mathrm{g} / \mathrm{kg}$ bodyweight (excluding continuous exposure in water for zebrafish), which is a thousand times higher than the TDI. All this puts into question whether the observed effects are truly specific to MC-LR exposure, or whether they represent a more general toxin response caused by toxin overload. 


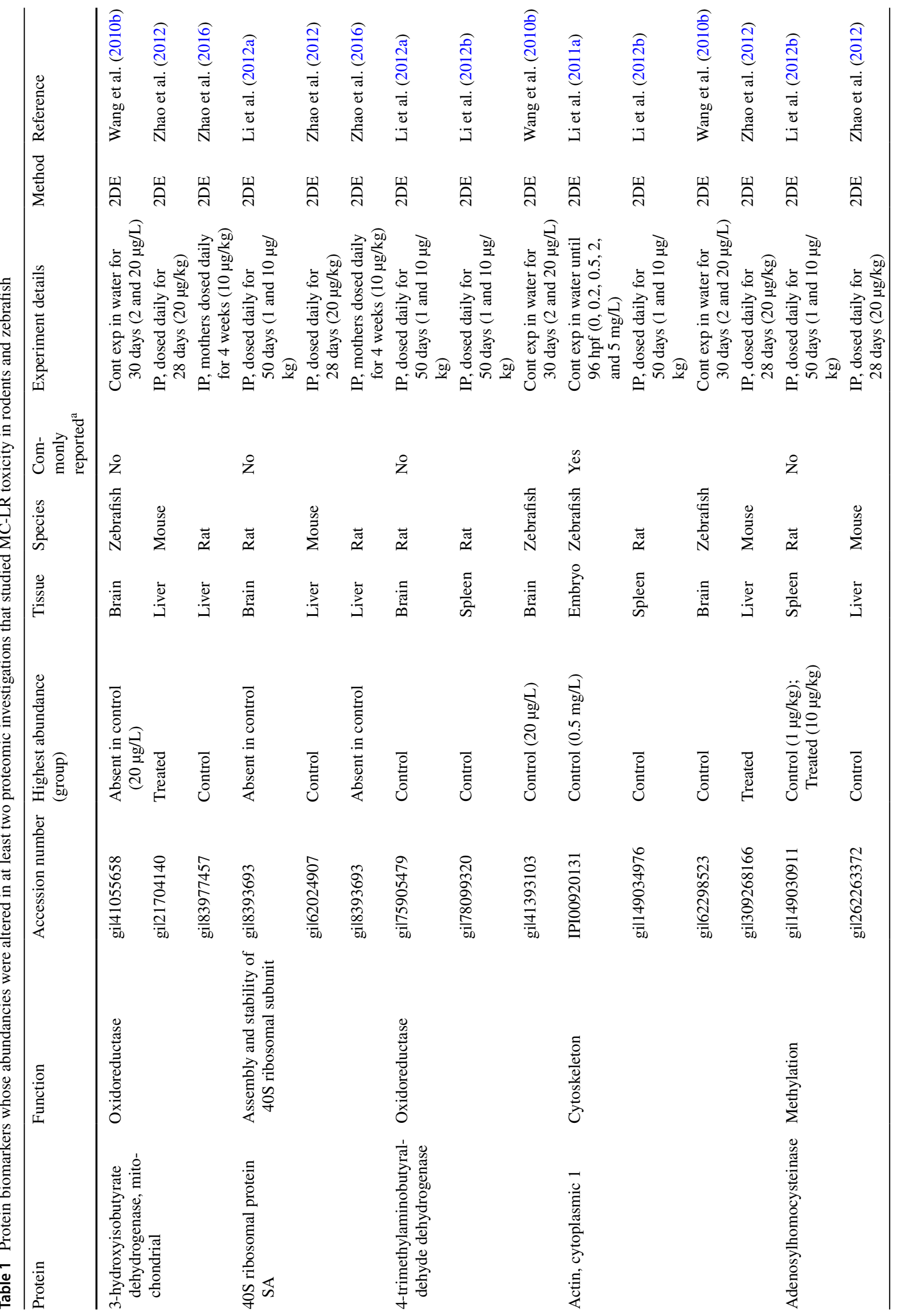




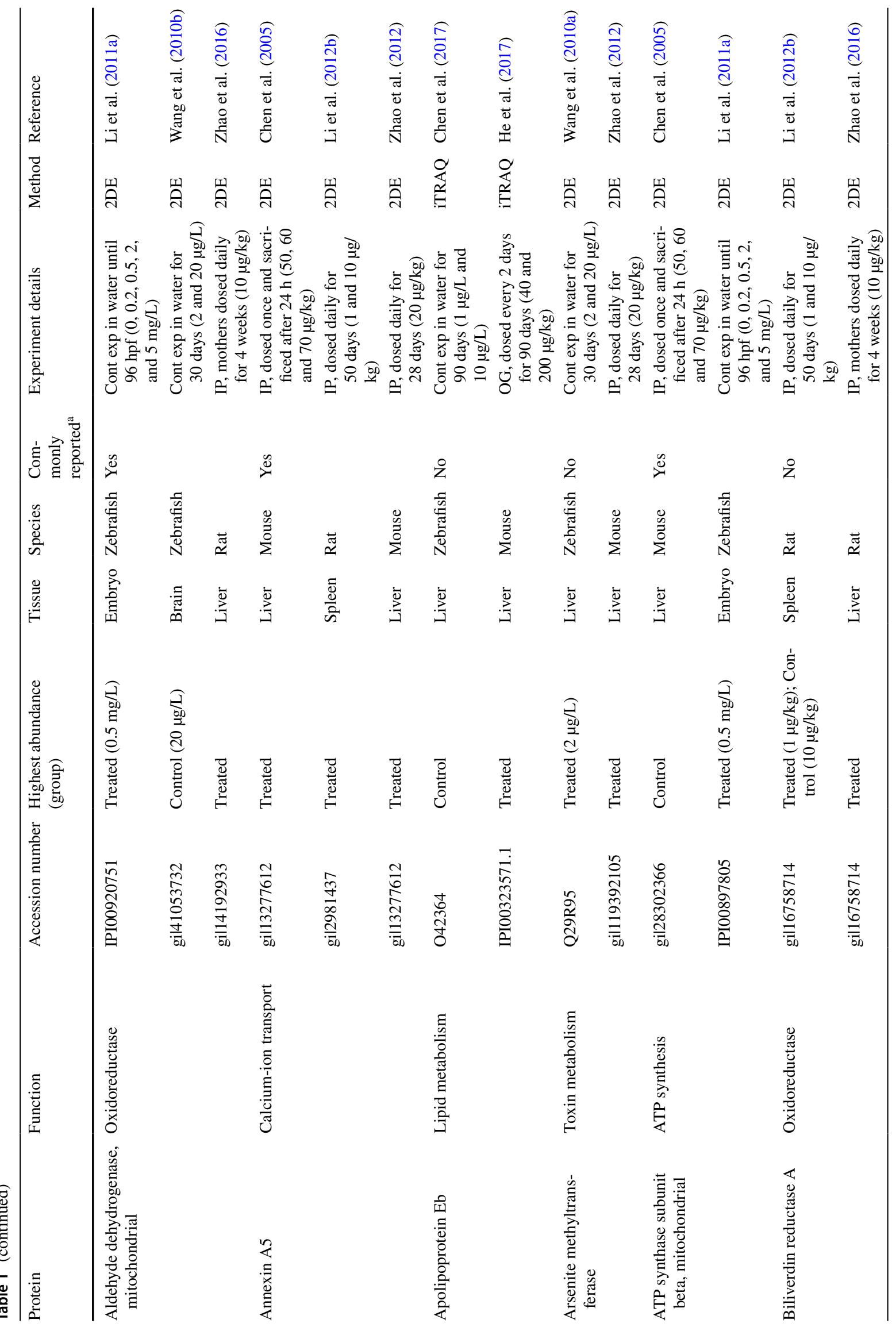




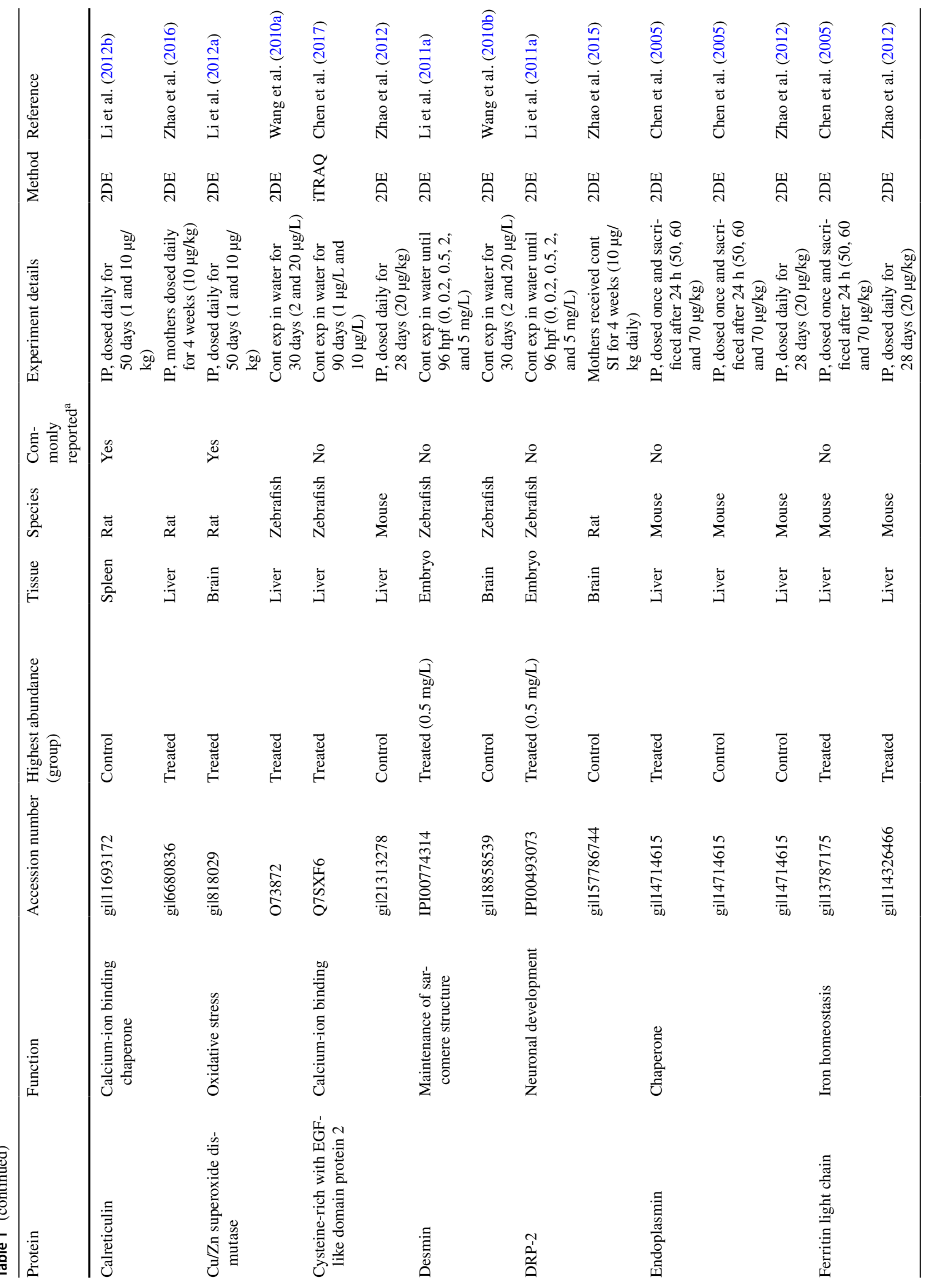




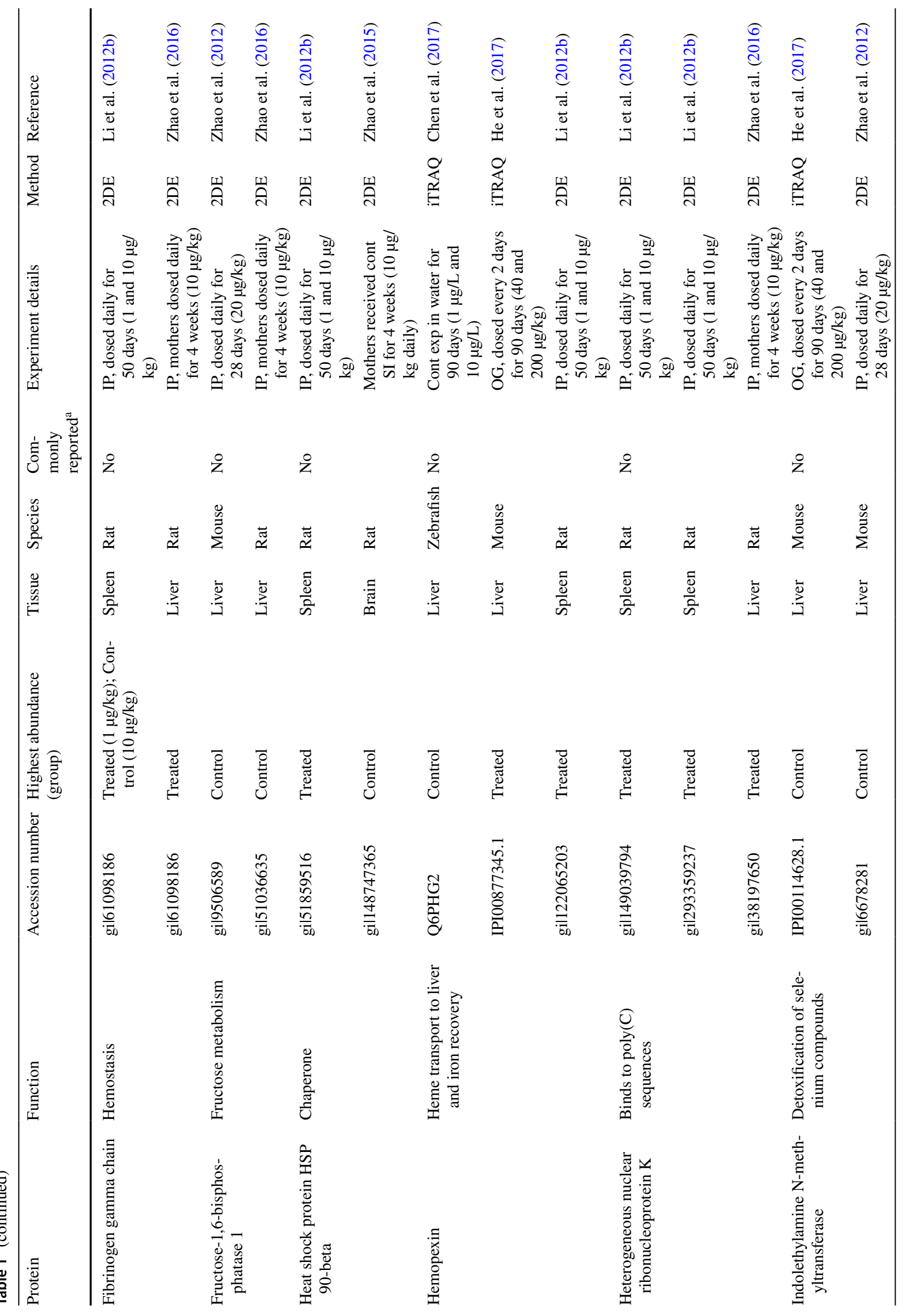




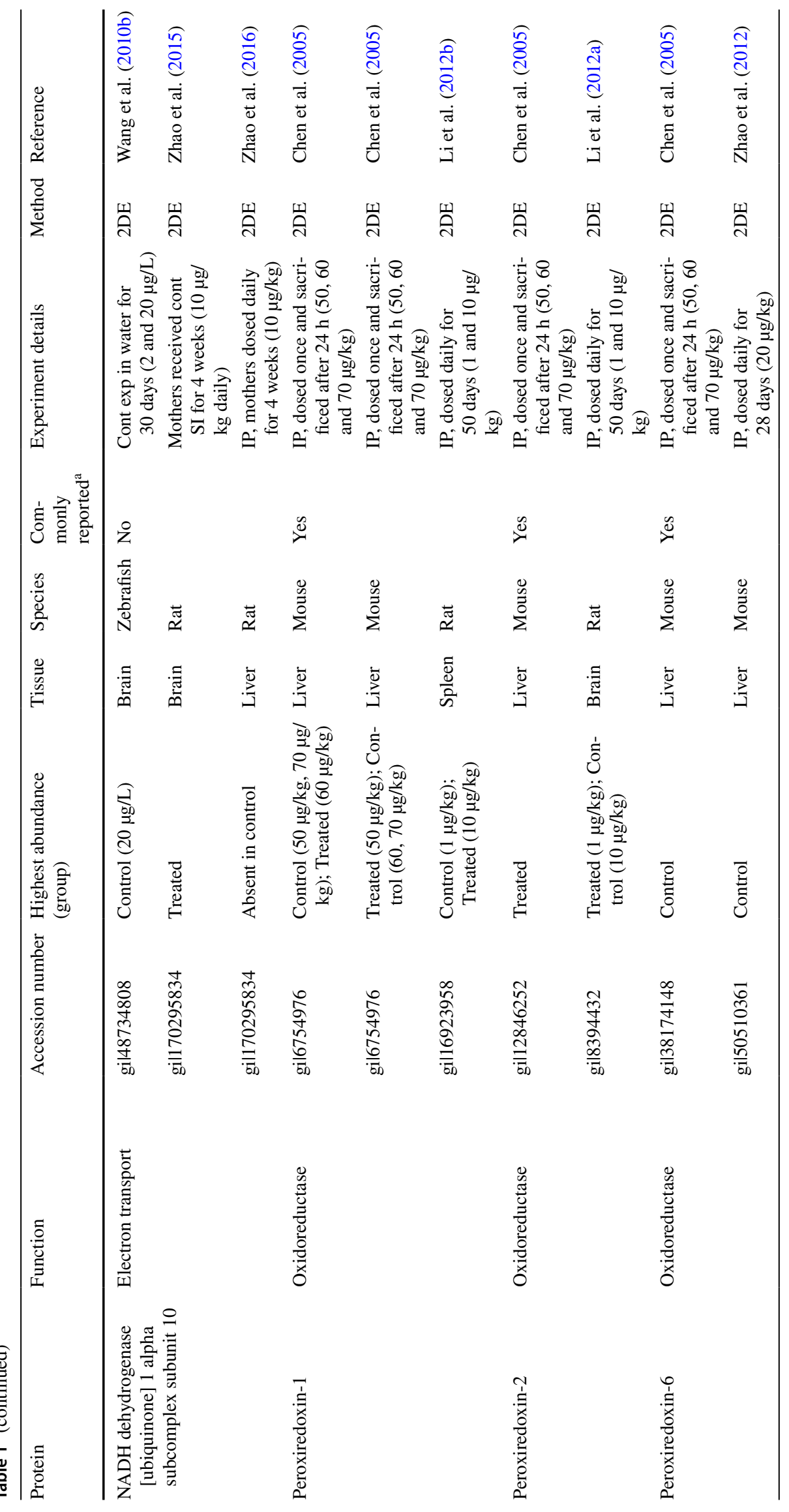




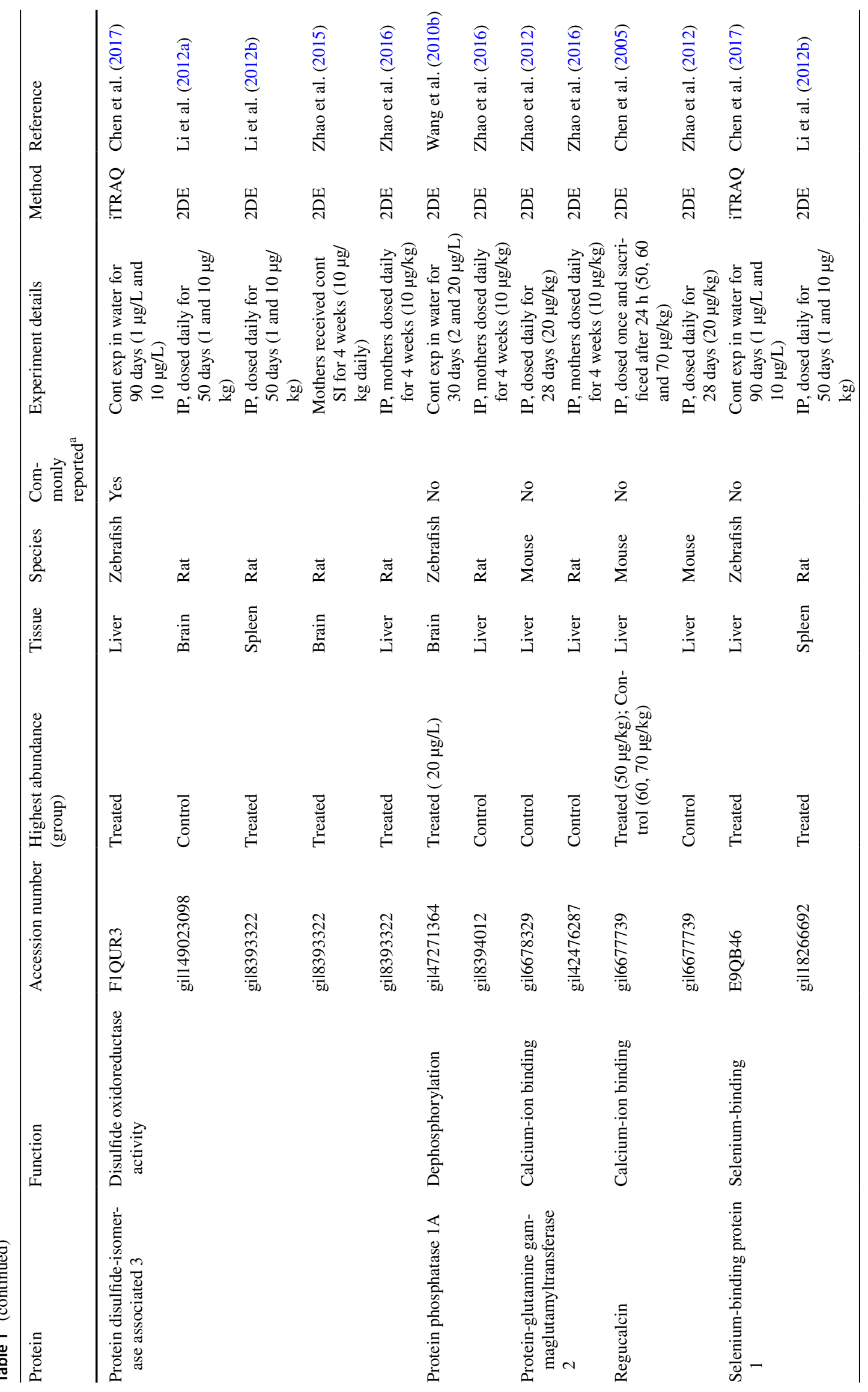




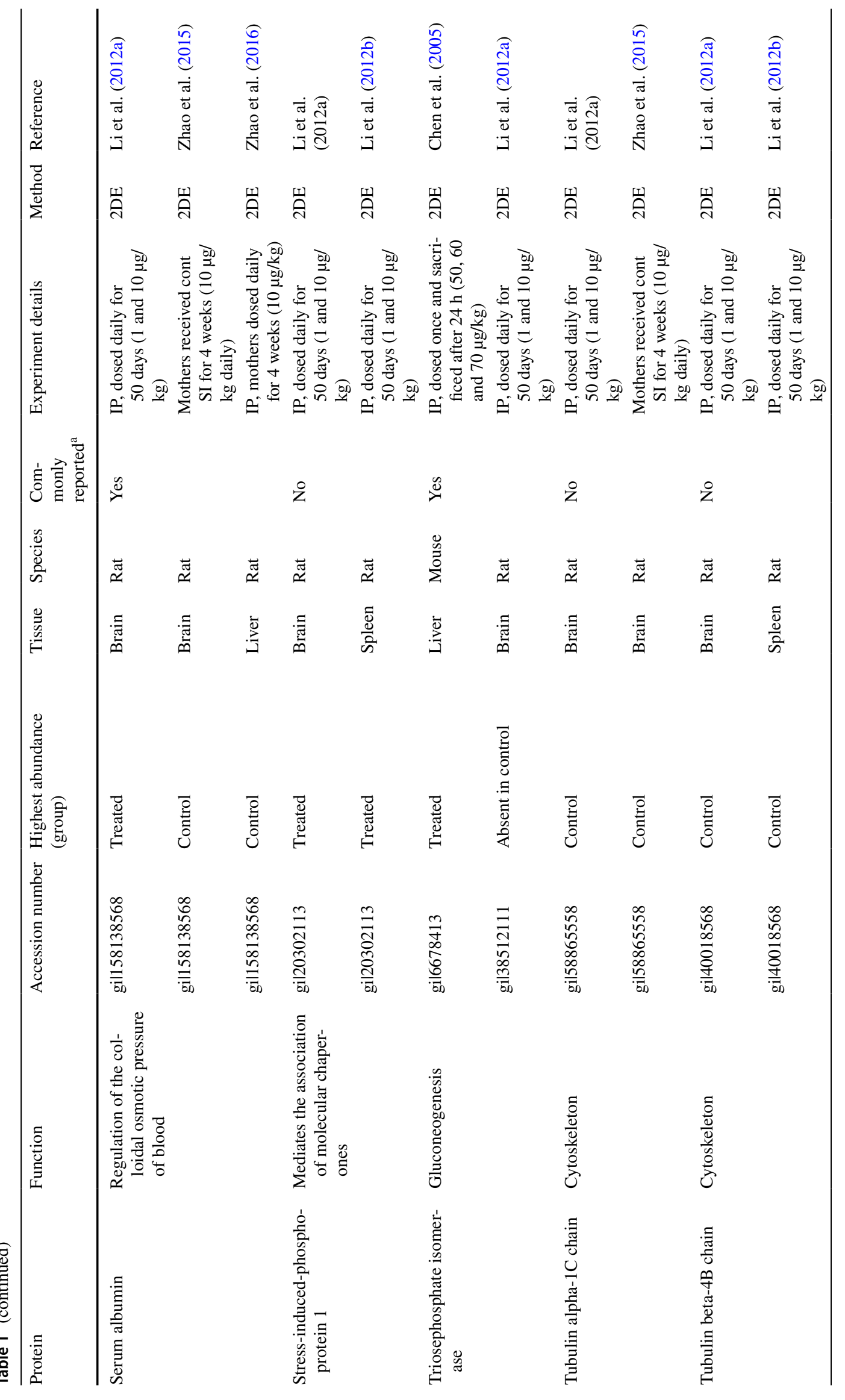




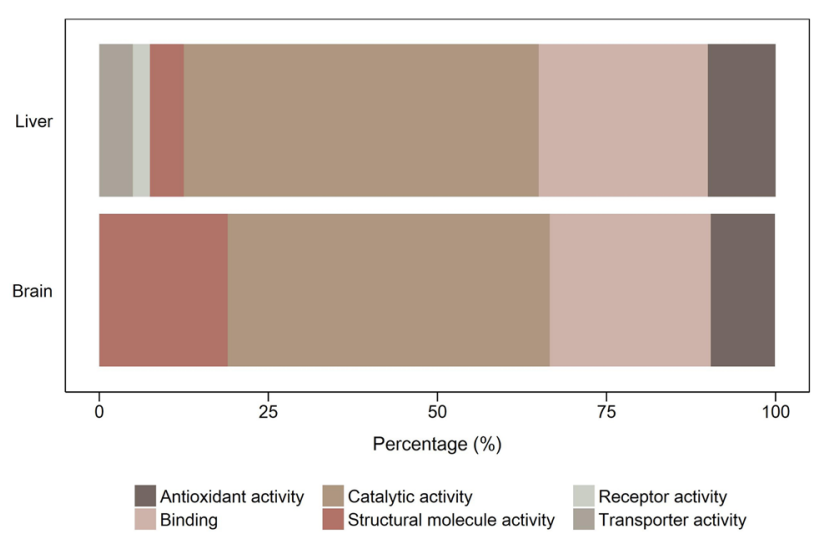

Fig. 1 Proportional representation of gene ontology molecular functions affected by MC-LR toxicity in liver and brain based on proteins whose abundancy levels changed in at least two proteomic studies (Table 1). In the liver, proteins affected by MC-LR were mainly involved in catalytic activity (52.5\%), binding (25.0\%), and antioxidant activity $(10.0 \%)$. As for the brain, there was an increase in structural molecule activity when compared to liver (19.0\% in brain, $5.0 \%$ in liver), whereas other overlapping categories remained largely the same

In fact, several studies found that certain proteins frequently exhibit abundancy changes in 2DE studies independent of the experimental conditions (Petrak et al. 2008; Wang et al. 2009; Mariman 2009). Petrak and colleagues established a list of top-15 generally detected proteins (Petrak et al. 2008), whereas a different study listed 44 proteins whose abundancies changed frequently across 2DE experiments (Wang et al. 2009). Table 1 indicates which potential MC-LR biomarkers overlap with the top 15 and top 44 of frequently reported proteins in 2DE analyses. Indeed, 12 proteins listed in Table 1 overlap with the frequently reported protein biomarkers, i.e., $30.8 \%$ of all proteins found in this review. Because these studies did not look at commonly reported proteins derived from 2DE analyses on zebrafish samples, this percentage may even be an underestimate, as it seems likely that certain zebrafish proteins could have the tendency to be detected more frequently than others, regardless of experimental setup.

The overrepresentation of certain proteins when using the 2DE method could have various reasons. It could be inherent to the 2DE technique allowing good visualization of proteins with certain properties, although this would not explain why their abundancies differ so frequently (Mariman 2009). In addition, cells are equipped with various stress response mechanisms that are phylogenetically conserved (Kultz 2003), and subtle changes in a cell's environment could trigger these (orthologous) pathways across multiple species. This could explain why certain proteins are repeatedly affected in many studies: they may represent a general and conserved stress response. Importantly, 
one shortcoming of the current proteomics literature on MC-LR toxicity is that nearly all work in mammals has been carried out using the 2DE technique. It would be interesting to see more studies exploring the effect of MC-LR on the proteome using different techniques, for example by employing shotgun proteomics or iTRAQ, to eliminate any possible bias generated by the 2DE method.

The large overlap of a vast number of proteins across multiple studies regardless of experimental conditions is problematic for data interpretation. This study attempted to tackle this issue by creating a list of proteins whose abundancies changed in at least two studies, and to flag those that are commonly represented in proteomic studies (Table 1), based on the top-15 and top-44 lists of frequently reported proteins in 2DE analyses (Petrak et al. 2008; Wang et al. 2009). This strategy increases the likelihood that the non-flagged proteins in Table 1 represent a specific stress response to MC-LR, but since the evidence is only circumstantial, more specific research on individual proteins would be required to confidently link an effect to MC-LR toxicity. The flagged proteins were nevertheless included in this review because while they may be reported commonly across many 2DE analyses and could therefore represent a more general stress response, they may still provide insights into MC-LR toxicity.

It should also be noted that it is difficult to compare a toxin's effect across multiple organisms (and tissues), as MC-LR may affect the zebrafish proteome differently than the rodent proteome. Nevertheless, it is reasonable to assume that similar cellular pathways are affected by MC-LR toxicity across different species and tissues. In addition, the limited number of proteomic studies on MC-LR toxicity performed per model organism make it difficult to effectively compare the published results for one species only. Due to these reasons, the choice was made to compare the proteomes of mice, rats, and zebrafish together, as these organisms have been employed most frequently in MC-LR proteomic studies.

To summarize, the goal of Table 1 is twofold. Firstly, it aims to connect proteomic studies investigating MC-LR toxicity in rodents and zebrafish to find common patterns in protein abundancy changes, and secondly to stimulate hypothesis generation.

\section{Overrepresented Molecular Functions}

The data derived from the overrepresentation tests show that proteins whose abundancies consistently changed after MC-LR exposure are often involved in antioxidant and oxidoreductase activity (Tables 2 and 3). This is in agreement with the widely supported theory that MC-LR induces oxidative stress by promoting reactive oxygen species (ROS) formation (Zegura 2016). For example, low concentrations of MC-LR induced double strand DNA breaks in HepG2 cells (Zegura et al. 2003). The same study found that digestion of
Table 2 Molecular functions overrepresented in proteomic studies that investigated MC-LR toxicity in the liver

\begin{tabular}{lclll}
\hline Molecular function & Observed & Expected & $p$ value $^{\mathrm{a}}$ & False discovery rate \\
\hline Peroxidase activity & 3 & 0.04 & $1.02 \mathrm{E}-05$ & $4.87 \mathrm{E}-04$ \\
Antioxidant activity & 4 & 0.05 & $3.47 \mathrm{E}-07$ & $3.33 \mathrm{E}-05$ \\
Oxidoreductase activity & 7 & 0.76 & $9.29 \mathrm{E}-06$ & $5.94 \mathrm{E}-04$ \\
Catalytic activity & 21 & 6.41 & $2.84 \mathrm{E}-08$ & $5.46 \mathrm{E}-06$ \\
\hline
\end{tabular}

${ }^{\text {a }} p$ values calculated by the PANTHER algorithm using the Fisher's exact test with FDR multiple test corrections. The gene ontology molecular function classes of the reported liver proteins were compared with the gene ontology molecular function classes of the Homo sapiens database (PANTHER release 13.1)

\begin{tabular}{lclll}
\hline Molecular function & Observed & Expected & $P$ value $^{\mathrm{a}}$ & False discovery rate \\
\hline Antioxidant activity & 2 & 0.03 & $4.51 \mathrm{E}-04$ & $4.33 \mathrm{E}-02$ \\
Structural constituent of cytoskeleton & 3 & 0.22 & $1.25 \mathrm{E}-03$ & $4.00 \mathrm{E}-02$ \\
Structural molecule activity & 4 & 0.43 & $7.88 \mathrm{E}-04$ & $5.04 \mathrm{E}-02$ \\
Oxidoreductase activity & 5 & 0.43 & $5.29 \mathrm{E}-05$ & $1.02 \mathrm{E}-02$ \\
Catalytic activity & 10 & 3.61 & $9.33 \mathrm{E}-04$ & $4.48 \mathrm{E}-02$ \\
\hline
\end{tabular}

${ }^{a} p$ values calculated by the PANTHER algorithm using the Fisher's exact test with FDR multiple test corrections. The gene ontology molecular function classes of the reported brain proteins were compared with the gene ontology molecular function classes of the Homo sapiens database (PANTHER release 13.1)
Table 3 Molecular functions overrepresented in proteomic studies that investigated MC-LR toxicity in the brain 
DNA with endonucleases specific for oxidative DNA damage increased DNA strand breaks in MC-LR-treated cells, indicating that oxidative stress is responsible. Genotoxicity by MC-LR has also been reported in human peripheral blood lymphocytes (Mankiewicz et al. 2002; Zegura et al. 2011), human colon adenocarcinoma cell line CaCo-2 (Zegura et al. 2008), and hamster ovary cells (Wang et al. 2013). Indeed, the data from Table 1 are consistent with the theory that MC-LR induces oxidative stress and may help to identify key players involved in this process that may not had been considered previously.

The results from the functional annotation analysis (Fig. 1) and overrepresentation tests (Tables 2 and 3) also indicate that proteins involved in cytoskeletal processes are affected by MC-LR, especially in the brain. There could be several reasons as of why the cytoskeleton appears more affected by MC-LR in the brain than in the liver. Since hepatocytes and neurons are different cell types, they could be affected differently by the toxin. Furthermore, MC-LR is transported into hepatocytes and across the blood brain barrier by organic-anion-transporting polypeptides (OATPs), which are membrane solute carriers that transport a wide range of amphipathic transport substrates (Hagenbuch and Meier 2003). In humans, members OATP1B1 and OATP1B3 transfer MC-LR across the cell membrane into hepatocytes (Komatsu et al. 2007), whereas OATP1A2 is responsible for MC-LR uptake through the blood-brain barrier (Fischer et al. 2005). Perhaps, MC-LR's use of different transporters leads to different exposure conditions in various organs. Additionally, MC-LR is thought to bioaccumulate at a higher rate in the liver than in the brain (Falconer et al. 1986; Wang et al. 2008), and perhaps these differences in toxin concentration lead to different cellular responses.

In general, the effects of MC-LR on the cytoskeleton could be a result of PP2A inhibition. Indeed, more than 30 protein kinase activities are regulated by PP2A in vitro (Millward et al. 1999). Some of these kinases are involved in cytoskeletal regulation, such as MAP kinase-activated protein kinase 2 (Millward et al. 1999; Zhou et al. 2015). It is plausible that MC-LR indirectly perturbs the cytoskeleton via PP2A inhibition. Because cytoskeleton and cytoskeletonassociated proteins are often involved in signal transduction (Wickstead and Gull 2011), MC-LR exposure could lead to disruptions within cell signaling pathways (Zhou et al. 2015), causing a wide range of complications. This may explain why proteins involved in an array of different functions have consistently been reported altered in multiple proteomic studies (Table 1).

To summarize, functional annotations (Fig. 1) and overrepresentation tests (Tables 2 and 3) suggest that MC-LR predominantly induces antioxidant activity in both the liver and brain, whereas in the brain the cytoskeleton appears more strongly affected than in the liver.

\section{Abundancy Changes of Individual Proteins}

While functional classification of proteins provides valuable information on the general mechanisms affected, it is also important to look individually at each protein influenced by MC-LR toxicity to determine in which studies a protein's level is consistently up-or downregulated. As mentioned previously, a subset of key cytoskeletal proteins shows recurring changes in abundancy across a number of proteomic studies. The observed trend is a general decrease in protein levels after MC-LR exposure (Table 1). These include chains of the microtubule protein tubulin, namely tubulin alpha-1C chain (Li et al. 2012a; Zhao et al. 2015) and tubulin beta4B chain ( $\mathrm{Li}$ et al. 2012a, b). Microtubules are polymers of tubulin dimers that form the spindle structure, which enables chromosome segregation during mitosis and meiosis (Gadde and Heald 2004). It was shown in CHO-K1 cells that MC-LR exposure leads to the formation of aberrant spindles (Lankoff et al. 2003). Cells treated with MC-LR showed abnormal anaphases with defective chromosome separation. This effect correlated with the formation of aberrant mitotic spindles, indicating that MC-LR exposure leads to the formation of dysfunctional spindles and thereby hinders cell division (Lankoff et al. 2003).

As can be seen in Table 1, levels of microfilament actin (cytoplasmic 1) also underwent a general decline after MC-LR exposure (Wang et al. 2010b; Li et al. 2011a, 2012b; Zhao et al. 2012). Multiple studies have shown that MC-LR causes microfilament disorganization (Zhou et al. 2015). For example, Batista and colleagues found that actin filaments collapsed in primary human hepatocytes treated with MC-LR (Batista et al. 2003). In a different study, primary rat hepatocytes underwent microfilament disorganization after MC-LR treatment (Ding et al. 2001). The authors also found an increase in ROS formation, and they suggested that the cytoskeleton disorganization may be a result of the increased presence of ROS. In conclusion, there is a substantial amount of evidence supporting the theory that MC-LR causes cytoskeletal disruption, which has been reviewed in more detail recently (Zhou et al. 2015). The data generated by multiple proteomic studies support the theory that MC-LR perturbs cytoskeletal processes.

As mentioned previously, MC-LR causes cellular disruption by inducing ROS formation. MC-LR therefore indirectly induces oxidative stress, a process known to cause genotoxicity. This subject has been reviewed in great detail recently (Zegura 2016). As can be seen in Table 1, numerous proteins involved in oxidoreductase activity and defense against oxidative stress were affected by MC-LR toxicity. These include members of the peroxiredoxin family, which are antioxidant enzymes that guard cells against oxidative stress and regulate intracellular dihydrogen peroxide $\left(\mathrm{H}_{2} \mathrm{O}_{2}\right)$ levels (Rhee et al. 2001). Peroxiredoxin-2 showed 
increased levels in both the rat hippocampus (Li et al. 2012a) and mouse liver (Chen et al. 2005) after MC-LR treatment (Table 1). While peroxiredoxin-2 regulates endogenous $\mathrm{H}_{2} \mathrm{O}_{2}$ signaling (Choi et al. 2005), it also protects metastatic cells in the lungs from oxidative stress (Stresing et al. 2013). Indeed, Stresing and colleagues found that lung metastatic variants of MDA-MB-435 breast cancer cells were less susceptible to oxidative stress, which correlated with increased levels of peroxiredoxin-2. Contrary to the peroxiredoxin-2 effects, two proteomic studies reported decreased quantities of peroxiredoxin- 6 after MC-LR treatment (Chen et al. 2005; Zhao et al. 2012), whereas levels of peroxiredoxin-1 were inconsistent across two studies (Chen et al. 2005; Li et al. 2012b). These variations could be due to the different regeneration mechanisms inherent to the peroxiredoxin family members (Chevallet et al. 2003). After oxidative stress, peroxiredoxin- 2 is the first to regenerate, while peroxiredoxin- 1 and peroxiredoxin- 6 regenerate more slowly (Chevallet et al. 2003). This indicates that while cells recover from oxidative stress, peroxiredoxin-2 levels increase at a much faster rate than the other peroxiredoxins, which could explain the variations found in peroxiredoxin levels after MC-LR treatment (Chen et al. 2005).

Another protein involved in ROS clean-up is $\mathrm{Cu} / \mathrm{Zn}$ superoxide dismutase, which showed increased quantities in two proteomic studies (Wang et al. 2010a; Li et al. 2012a). Superoxide dismutases form the first line of defense against ROS, superoxides in particular (Zelko et al. 2002). They contain a metal ion cofactor that allows them to add or remove an electron from the superoxide and convert it into less toxic oxygen and $\mathrm{H}_{2} \mathrm{O}_{2}$ (Culotta et al. 2006).

Importantly, it should be noted that peroxiredoxin and $\mathrm{Cu} / \mathrm{Zn}$ superoxide dismutase levels are often changed in proteome studies regardless of experimental setup (Petrak et al. 2008; Wang et al. 2009). This means that these effects could simply represent a general toxin response and may not be specific for MC-LR toxicity.

Levels of ferritin light chain, a subunit of the ferritin protein, also increased after MC-LR exposure in two proteomic studies (Chen et al. 2005; Zhao et al. 2012). Ferritin is involved in iron (Fe) storage by binding $\mathrm{Fe}(\mathrm{II})$ in its ferroxidase center, where either $\mathrm{H}_{2} \mathrm{O}_{2}$ or oxygen are used to oxidize Fe(II) to Fe(III) (Arosio and Levi 2002; Arosio et al. 2009). The iron then migrates to the ferritin cavity where it nucleates and forms the iron core. This process allows the concentration and safe storage of up to 4000 iron atoms, which are readily available when required. Importantly, it prevents the spontaneous oxidation of $\mathrm{Fe}(\mathrm{II})$ to $\mathrm{Fe}(\mathrm{III})$, a reaction that generates ROS (Arosio et al. 2009). Indeed, ferritin prevents free radical production by isolating $\mathrm{Fe}(\mathrm{II})$ atoms and thereby guards the cell against oxidative stress. Since MC-LR induces the formation of ROS, perhaps the cell responds by generating more ferritin to clear other sources of ROS formation from the cell.

Levels of heterogeneous nuclear ribonucleoprotein $\mathrm{K}$ (HNRNPK) were increased in two proteomic studies ( $\mathrm{Li}$ et al. 2012b; Zhao et al. 2016). HNRNPK is a transcriptional cofactor of $\mathrm{p} 53$, a widely studied transcription factor known for its tumor-suppressing capabilities (Moumen et al. 2005). Also known as the "guardian of the genome", p53 is involved in many cellular activities, including DNA repair and defense against oxidative stress (Nicolai et al. 2015). Perhaps cells produce more HNRNPK to activate defense mechanisms through p53 in order to fight oxidative stress caused by MC-LR toxicity.

Two studies reported an increased abundance of selenium-binding protein 1 (Li et al. 2012b; Chen et al. 2017), which covalently binds selenium and participates in intraGolgi protein transport (Porat et al. 2000; Jeong et al. 2009). It also takes part in toxification and detoxification processes (Papp et al. 2007). Interestingly, selenium supplementation prior to MC-LR administration provided some protection against hepatotoxicity in mice (Gehringer et al. 2003). Indeed, selenium pretreatment resulted in less severe liver injury, as well as decreased serum ALT and lipid peroxidation levels compared to rodents that received MC-LR alone. The proteomics data suggest that selenium pathways play a role in MC-LR toxicity. It may be interesting to research this finding in more detail, especially as selenium supplementation has been reported to guard against MC-LR toxicity (Gehringer et al. 2003).

\section{Human Health Implications}

Overall, the proteomic studies investigating MC-LR toxicity in rodents and zebrafish have illustrated that a wide array of molecular functions are affected. However, these experiments have been performed in animals under varying experimental conditions that do not allow easy comparison, and thus the key question remains what these results mean in terms of human exposure to MC-LR.

Importantly, it should be pointed out that the toxin concentrations used in all discussed proteomic studies were extremely high (Table 1). The lowest oral dose applied in mammals was $40 \mu \mathrm{g} / \mathrm{kg}$ bodyweight (He et al. 2017), or a thousand times the TDI. To put this into perspective, fishermen who had been living for at least five years on a lake regularly plagued by cyanobacterial blooms were estimated to consume a daily amount of 2.2 to $3.9 \mu \mathrm{g}$ of MC-LR equivalents per person (Chen et al. 2009). For a person weighing $60 \mathrm{~kg}$, this roughly translates to 0.037 to $0.065 \mu \mathrm{g} / \mathrm{kg}$ bodyweight, and thus the estimated intake of these fishermen was close to the TDI of $0.04 \mu \mathrm{g} / \mathrm{kg}$ bodyweight set by the WHO. The fishermen would thus need to increase their daily MC-LR intake roughly a thousand times in order to meet 
the lowest applied dose in mammals in a proteomic study ( $40 \mu \mathrm{g} / \mathrm{kg}$ bodyweight), and this puts into question how the results translate to human exposure conditions. The timeframes applied in these proteomic studies were often relatively short (Table 1), not exceeding three months, whereas the fishermen had been living on the lake for at least five years. This reveals two complications in the current MC-LR proteomics literature that make it difficult to translate these results into a human scenario. Firstly, the MC-LR concentrations dosed are extremely high, and secondly, the experiment durations are relatively short.

Interestingly, a non-proteomic study did look at the effect of low dose MC-LR toxicity on mice over several periods of months, the longest being 18 months (Ueno et al. 1999). The animals received $20 \mu \mathrm{g} \mathrm{MC}-\mathrm{LR} / \mathrm{L}$ in their drinking water, which translates to twenty times the guideline value of $1 \mu \mathrm{g} / \mathrm{L}$ set by the WHO for MC-LR in drinking water (Chorus and Bartram 1999). The authors looked at hematology and serum biochemistry parameters, as well as histopathology of various organs. No signs of chronic toxicity were found and no MC-LR was detected in the livers. Importantly, these results derive from an experiment that more closely mimicked exposure conditions relevant to humans, and they provide no proof that MC-LR is toxic under these experimental conditions. Unfortunately, the authors did not look at the proteome, which perhaps would have allowed detection of more subtle changes in protein levels in response to MC-LR exposure.

As mentioned previously, the findings reported in this review reinforce the theory that MC-LR induces oxidative stress, a process implicated with carcinogenesis (Klaunig and Kamendulis 2004). It should also be noted that while oxidative stress markers were regularly found affected in the proteome studies, these effects could also reflect a general stress response and may not have a role in carcinogenesis. Nevertheless, the finding correlates with the fact that several epidemiology studies have linked MC-LR exposure to increased incidences of cancer. For example, a positive correlation was found between the incidence rate of colorectal cancer and people who used water from ponds and rivers that contained microcystins, compared to those who used well and tap water (Lun et al. 2002). In addition, Ueno and colleagues found a correlation between microcystin levels in drinking water and the incidence of primary liver cancer in Haimen city, China (Ueno et al. 1996). In the same article, however, the authors write that major foodstuffs in Haimen City contained aflatoxin B1, a carcinogen known to affect the liver (Bennett and Klich 2003). This illustrates the difficulty of interpreting results from epidemiology studies, as they only indicate correlations, but no direct proof. In all epidemiology studies investigating MC-LR toxicity, the participants could have been exposed to a wide array of toxins present in their water sources, making it extremely challenging to pinpoint MC-LR as the (sole) contributor of the measured effects. This further stresses the need for animal studies that better mimic human exposure conditions, preferably ones that take a systems biology approach to study different levels of biological organization.

To summarize, it would be interesting to study the effect of chronic low-dose MC-LR exposure on the proteome over a longer period of time, thus better portraying a realistic human scenario. The findings in Table 1 help highlight proteins whose abundancies changed in response to MC-LR toxicity in at least two studies, however, the question remains whether they would also be affected in humans that are chronically exposed to low MC-LR amounts. Indeed, more proteomic studies accurately portraying human exposure conditions are needed to better understand how the human proteome could be affected.

\section{Conclusion}

A large number of studies have looked at the effect of MC-LR toxicity on the proteome, generating a substantial list of potential biomarkers. It is difficult to determine which of the observed effects are MC-LR specific. Indeed, some proteins may show altered levels due to a general toxin response, whereas others may be influenced by other factors (e.g., experimental design). However, a protein's response is more likely to be caused by the toxin if the results are repeated in more than one proteomic study. This review identified 39 proteins that were affected in at least two proteomic studies investigating MC-LR toxicity and flagged those that are commonly found in 2DE analyses regardless of experimental setup (Table 1).

One consistent effect reported by proteomic studies is the decreased levels of cytoskeletal components after MC-LR exposure. These include tubulin microtubules and actin microfilaments (Table 1). The adverse effects of MC-LR on the cytoskeleton have been reviewed in detail previously (Zhou et al. 2015), and this review helps identify the possible key players involved. Evidence suggests that MC-LR induces oxidative stress, which leads to genotoxicity and possibly carcinogenicity (Zegura 2016). Further studies on the proteins listed in Table 1 may shed more light on the genotoxic and possible carcinogenic effects of MC-LR. Other proteins whose levels were perturbed by MC-LR include those that play a role in calcium binding, protein folding, and metabolism, which could be due to PP2A regulating a wide range of cell signaling pathways (Millward et al. 1999). Indeed, the proteins from Table 1 illustrate that MC-LR exposure affects many components of the cellular machinery.

However, it should be pointed out that the available data are limited, and it is difficult to adequately compare results 
from multiple species and tissue types. Because of the extreme experimental conditions applied (high toxin doses and relatively short timeframes), it is challenging to translate these results into a human exposure scenario. More data from MC-LR proteomic studies, preferably performed in mammals using low oral MC-LR concentrations, would help shed more light onto the effect of MC-LR on the proteome. A low oral dose is key as it would better represent human exposure conditions. Furthermore, because MS-based methods such as shotgun proteomics and iTRAQ are underrepresented in the current literature, it would be a valuable addition to explore the effect of MC-LR on the proteome using these techniques.

To conclude, this review exposes consistently reported proteins affected by MC-LR exposure that may not had been considered previously, opening new doors to understanding the mechanisms behind MC-LR toxicity. Additional research will be required to verify these results.

Funding The authors would like to thank the Department of Employment and Learning (DEL) for funding the project: "Metabolomic and array based biomarker approaches to understand human exposure to potent carcinogenic fresh water toxins" (reference, 14/IA/2646) via the Science Foundation Ireland (SFI)-DEL Investigators Programme Partnership.

\section{Compliance with ethical standards}

Conflict of interest The authors declare that they have no conflict of interest.

Open Access This article is distributed under the terms of the Creative Commons Attribution 4.0 International License (http://creativeco mmons.org/licenses/by/4.0/), which permits unrestricted use, distribution, and reproduction in any medium, provided you give appropriate credit to the original author(s) and the source, provide a link to the Creative Commons license, and indicate if changes were made.

\section{References}

van Apeldoorn ME, van Egmond HP, Speijers GJA, Bakker GJI (2007) Toxins of cyanobacteria. Mol Nutr Food Res 51:7-60. https://doi. org/10.1002/mnfr.200600185

Arosio P, Levi S (2002) Ferritin, iron homeostasis, and oxidative damage. Free Radic Biol Med 33:457-463. https://doi.org/10.1016/ S0891-5849(02)00842-0

Arosio P, Ingrassia R, Cavadini P (2009) Ferritins: A family of molecules for iron storage, antioxidation and more. Biochim Biophys Acta BBA 1790:589-599. https://doi.org/10.1016/j.bbage n.2008.09.004

Batista T, de Sousa G, Suput JS et al (2003) Microcystin-LR causes the collapse of actin filaments in primary human hepatocytes. Aquat Toxicol 65:85-91. https://doi.org/10.1016/S0166-445X(03)00108 $-5$

Bennett JW, Klich M (2003) Mycotoxins. Clin Microbiol Rev 16:497516. https://doi.org/10.1128/CMR.16.3.497-516.2003
Carmichael WW, An JS, Azevedo S et al (1996) Analysis for microcystins involved in outbreak of liver failure and death of humans at a hemodialysis center in Caruaru, Pernambuco. Brazil, IV Simpósio Soc Bras Toxinologia

Chen T, Wang Q, Cui J et al (2005) Induction of apoptosis in mouse liver by microcystin-LR a combined transcriptomic, proteomic, and simulation strategy. Mol Cell Proteomics 4:958-974. https ://doi.org/10.1074/mcp.M400185-MCP200

Chen J, Xie P, Li L, Xu J (2009) First identification of the hepatotoxic microcystins in the serum of a chronically exposed human population together with indication of hepatocellular damage. Toxicol Sci 108:81-89. https://doi.org/10.1093/toxsci/kfp009

Chen $\mathrm{L}, \mathrm{Hu} \mathrm{Y}, \mathrm{He} \mathrm{J}$ et al (2017) Responses of the proteome and metabolome in livers of zebrafish exposed chronically to environmentally relevant concentrations of microcystin-LR. Environ Sci Technol 51:596-607. https://doi.org/10.1021/acs.est.6b039 90

Chevallet M, Wagner E, Luche S et al (2003) Regeneration of peroxiredoxins during recovery after oxidative stress: only some overoxidized peroxiredoxins can be reduced during recovery after oxidative stress. J Biol Chem 278:37146-37153. https://doi. org/10.1074/jbc.M305161200

Choi MH, Lee IK, Kim GW et al (2005) Regulation of PDGF signalling and vascular remodelling by peroxiredoxin II. Nature 435:347-353. https://doi.org/10.1038/nature03587

Chorus I, Bartram J (1999) Toxic cyanobacteria in water: a guide to their public health consequences, monitoring, and management. E \& FN Spon, London

Codd GA, Metcalf JS, Beattie KA (1999) Retention of Microcystis aeruginosa and microcystin by salad lettuce (Lactuca sativa) after spray irrigation with water containing cyanobacteria. Toxicon 37:1181-1185

Culotta VC, Yang M, O'Halloran TV (2006) Activation of superoxide dismutases: putting the metal to the pedal. Biochim Biophys Acta 1763:747-758. https://doi.org/10.1016/j.bbamcr.2006.05.003

Ding W-X, Shen H-M, Ong C-N (2001) Critical role of reactive oxygen species formation in microcystin-induced cytoskeleton disruption in primary cultured hepatocytes. J Toxicol Environ Health A 64:507-519. https://doi.org/10.1080/152873901753215966

Falconer IR (2005) Is there a human health hazard from microcystins in the drinking water supply? Acta Hydrochim Hydrobiol 33:64-71. https://doi.org/10.1002/aheh.200300551

Falconer IR, Beresford AM, Runnegar MT (1983) Evidence of liver damage by toxin from a bloom of the blue-green alga, Microcystis aeruginosa. Med J Aust 1:511-514

Falconer IR, Buckley T, Runnegar MT (1986) Biological half-life, organ distribution and excretion of 125-I-labelled toxic peptide from the blue-green alga Microcystis aeruginosa. Aust J Biol Sci 39:17-21

Fawell JK, Mitchell RE, Everett DJ, Hill RE (1999) The toxicity of cyanobacterial toxins in the mouse: I microcystin-LR. Hum Exp Toxicol 18:162-167. https://doi.org/10.1177/096032719901800 305

Fischer WJ, Altheimer S, Cattori V et al (2005) Organic anion transporting polypeptides expressed in liver and brain mediate uptake of microcystin. Toxicol Appl Pharmacol 203:257-263. https://doi. org/10.1016/j.taap.2004.08.012

Gadde S, Heald R (2004) Mechanisms and molecules of the mitotic spindle. Curr Biol 14:R797-R805. https://doi.org/10.1016/j. cub.2004.09.021

Garcia A, Cayla X, Guergnon J et al (2003) Serine/threonine protein phosphatases PP1 and PP2A are key players in apoptosis. Biochimie 85:721-726. https://doi.org/10.1016/j.biochi.2003.09.004

Gehringer MM (2004) Microcystin-LR and okadaic acid-induced cellular effects: a dualistic response. FEBS Lett 557:1-8. https://doi. org/10.1016/S0014-5793(03)01447-9 
Gehringer MM, Downs KS, Downing TG et al (2003) An investigation into the effect of selenium supplementation on microcystin hepatotoxicity. Toxicon 41:451-458. https://doi.org/10.1016/S0041 -0101(02)00362-8

Hagenbuch B, Meier PJ (2003) The superfamily of organic anion transporting polypeptides. Biochim Biophys Acta BBA - Biomembr 1609:1-18. https://doi.org/10.1016/S0005-2736(02)00633-8

He J, Li G, Chen J et al (2017) Prolonged exposure to low-dose microcystin induces nonalcoholic steatohepatitis in mice: a systems toxicology study. Arch Toxicol 91:465-480. https://doi.org/10.1007/ s00204-016-1681-3

Heinze R (1999) Toxicity of the cyanobacterial toxin microcystin-LR to rats after 28 days intake with the drinking water. Environ Toxicol 14:57-60. https://doi.org/10.1002/(SICI)1522-7278(19990 2)14:1\%3c57:AID-TOX9\%3e3.0.CO;2-J

Jeong J-Y, Wang Y, Sytkowski AJ (2009) Human selenium binding protein-1 (hSP56) interacts with VDU1 in a selenium-dependent manner. Biochem Biophys Res Commun 379:583-588. https:// doi.org/10.1016/j.bbrc.2008.12.110

Jochimsen EM, Carmichael WW, An J et al (1998) Liver Failure and Death after Exposure to Microcystins at a Hemodialysis Center in Brazil. N Engl J Med 338:873-878. https://doi.org/10.1056/ NEJM199803263381304

Klaunig JE, Kamendulis LM (2004) The Role of Oxidative Stress in Carcinogenesis. Annu Rev Pharmacol Toxicol 44:239-267. https ://doi.org/10.1146/annurev.pharmtox.44.101802.121851

Komatsu M, Furukawa T, Ikeda R et al (2007) Involvement of Mitogen-Activated Protein Kinase Signaling Pathways in MicrocystinLR-Induced Apoptosis after its Selective Uptake Mediated by OATP1B1 and OATP1B3. Toxicol Sci 97:407-416. https://doi. org/10.1093/toxsci/kfm054

Kultz D (2003) Evolution of the cellular stress proteome: from monophyletic origin to ubiquitous function. J Exp Biol 206:3119-3124. https://doi.org/10.1242/jeb.00549

Lance E, Neffling M-R, Gérard C et al (2010) Accumulation of free and covalently bound microcystins in tissues of Lymnaea stagnalis (Gastropoda) following toxic cyanobacteria or dissolved microcystin-LR exposure. Environ Pollut 158:674-680. https:// doi.org/10.1016/j.envpol.2009.10.025

Lankoff A, Banasik A, Obe G et al (2003) Effect of microcystin-LR and cyanobacterial extract from polish reservoir of drinking water on cell cycle progression, mitotic spindle, and apoptosis in CHO-K1 cells. Toxicol Appl Pharmacol 189:204-213. https://doi. org/10.1016/S0041-008X(03)00094-2

Li G, Chen J, Xie P et al (2011a) Protein expression profiling in the zebrafish (Danio rerio) embryos exposed to the microcystin-LR. Proteomics 11:2003-2018. https://doi.org/10.1002/pmic.20100 0442

Li Y, Chen J, Zhao Q et al (2011b) A cross-sectional investigation of chronic exposure to microcystin in relationship to childhood liver damage in the Three Gorges Reservoir Region, China. Environ Health Perspect 119:1483-1488. https://doi.org/10.1289/ ehp. 1002412

Li G, Cai F, Yan W et al (2012a) A proteomic analysis of MCLRinduced neurotoxicity: Implications for Alzheimer's disease. Toxicol Sci 127:485-495. https://doi.org/10.1093/toxsci/kfs114

Li G, Yan W, Qiao Q et al (2012b) Global effects of subchronic treatment of microcystin-LR on rat splenetic protein levels. J Proteomics 77:383-393. https://doi.org/10.1016/j.jprot.2012.09.012

Lun Z, Hai Y, Kun C (2002) Relationship between microcystin in drinking water and colorectal cancer. Biomed Env Sci 15:166-171

Magalhães V, Marinho M, Domingos P et al (2003) Microcystins (cyanobacteria hepatotoxins) bioaccumulation in fish and crustaceans from Sepetiba Bay (Brasil, RJ). Toxicon 42:289-295. https ://doi.org/10.1016/S0041-0101(03)00144-2
Mankiewicz J, Walter Z, Tarczynska M et al (2002) Genotoxicity of cyanobacterial extracts containing microcystins from Polish water reservoirs as determined by SOS chromotest and comet assay. Environ Toxicol 17:341-350. https://doi.org/10.1002/tox.10061

Mariman EC (2009) 2DE-proteomics meta-data indicate the existence of distinct cellular stress-responsive mechanisms. Expert Rev Proteomics 6:337-339. https://doi.org/10.1586/epr.09.50

Meneely JP, Elliott CT (2013) Microcystins: measuring human exposure and the impact on human health. Biomarkers 18:639-649. https://doi.org/10.3109/1354750X.2013.841756

Mi H, Muruganujan A, Casagrande JT, Thomas PD (2013) Large-scale gene function analysis with the PANTHER classification system. Nat Protoc 8:1551-1566. https://doi.org/10.1038/nprot.2013.092

Millward TA, Zolnierowicz S, Hemmings BA (1999) Regulation of protein kinase cascades by protein phosphatase $2 \mathrm{~A}$. Trends Biochem Sci 24:186-191. https://doi.org/10.1016/S0968-0004(99)01375-4

Mohamed ZA, Al Shehri AM (2009) Microcystins in groundwater wells and their accumulation in vegetable plants irrigated with contaminated waters in Saudi Arabia. J Hazard Mater 172:310 315. https://doi.org/10.1016/j.jhazmat.2009.07.010

Mohamed ZA, Carmichael WW, Hussein AA (2003) Estimation of microcystins in the freshwater fishOreochromis niloticus in an Egyptian fish farm containing aMicrocystis bloom. Environ Toxicol 18:137-141. https://doi.org/10.1002/tox.10111

Moumen A, Masterson P, O'Connor MJ, Jackson SP (2005) hnRNP $\mathrm{K}$ : An HDM2 target and transcriptional coactivator of p53 in response to DNA damage. Cell 123:1065-1078. https://doi. org/10.1016/j.cell.2005.09.032

Nicolai S, Rossi A, Di Daniele N et al (2015) DNA repair and aging: the impact of the p53 family. Aging 7:1050-1065. https://doi. org/10.18632/aging.100858

Papp LV, Lu J, Holmgren A, Khanna KK (2007) From selenium to selenoproteins: synthesis, identity, and their role in human health. Antioxid Redox Signal 9:775-806. https://doi.org/10.1089/ ars.2007.1528

Petrak J, Ivanek R, Toman O et al (2008) Déjà vu in proteomics. A hit parade of repeatedly identified differentially expressed proteins. Proteomics 8:1744-1749. https://doi.org/10.1002/pmic.20070 0919

Pitois S, Jackson MH, Wood BJ (2001) Sources of the eutrophication problems associated with toxic algae: an overview. J Environ Health 64:25-32

Porat A, Sagiv Y, Elazar Z (2000) A 56-kDa selenium-binding protein participates in intra-Golgi protein transport. J Biol Chem 275:14457-14465. https://doi.org/10.1074/jbc.275.19.14457

Pouria S, de Andrade A, Barbosa J et al (1998) Fatal microcystin intoxication in haemodialysis unit in Caruaru, Brazil. Lancet Lond Engl 352:21-26

Rhee SG, Kang SW, Chang T-S et al (2001) Peroxiredoxin, a novel family of peroxidases. IUBMB Life 52:35-41. https://doi. org/10.1080/15216540252774748

Runnegar M, Berndt N, Kong SM et al (1995) In vivo and in vitro binding of microcystin to protein phosphatases 1 and 2A. Biochem Biophys Res Commun 216:162-169. https://doi.org/10.1006/ bbrc. 1995.2605

Sedan D, Laguens M, Copparoni G et al (2015) Hepatic and intestine alterations in mice after prolonged exposure to low oral doses of microcystin-LR. Toxicon 104:26-33. https://doi.org/10.1016/j. toxicon.2015.07.011

Stresing V, Baltziskueta E, Rubio N et al (2013) Peroxiredoxin 2 specifically regulates the oxidative and metabolic stress response of human metastatic breast cancer cells in lungs. Oncogene 32:724735. https://doi.org/10.1038/onc. 2012.93

Ueno Y, Nagata S, Tsutsumi T et al (1996) Detection of microcystins, a blue-green algal hepatotoxin, in drinking water sampled in Haimen and Fusui, endemic areas of primary liver 
cancer in China, by highly sensitive immunoassay. Carcinogenesis 17:1317-1321

Ueno Y, Makita Y, Nagata S et al (1999) No chronic oral toxicity of a low dose of microcystin-LR, a cyanobacterial hepatotoxin, in female BALB/c mice. Environ Toxicol 14:45-55. https://doi.org/10.1002/(SICI) 1522-7278(19990 2) $14: 1 \% 3 c 45: A I D-T O X 8 \% 3 e 3.0 . C O ; 2-T$

Wang Q, Xie P, Chen J, Liang G (2008) Distribution of microcystins in various organs (heart, liver, intestine, gonad, brain, kidney and lung) of Wistar rat via intravenous injection. Toxicon 52:721-727. https://doi.org/10.1016/j.toxicon.2008.08.004

Wang P, Bouwman FG, Mariman ECM (2009) Generally detected proteins in comparative proteomics - A matter of cellular stress response? Proteomics 9:2955-2966. https://doi.org/10.1002/ pmic. 200800826

Wang M, Chan LL, Si M et al (2010a) Proteomic analysis of hepatic tissue of zebrafish (Danio rerio) experimentally exposed to chronic microcystin-LR. Toxicol Sci 113:60-69. https://doi.org/10.1093/ toxsci/kfp248

Wang M, Wang D, Lin L, Hong H (2010b) Protein profiles in zebrafish (Danio rerio) brains exposed to chronic microcystin-LR. Chemosphere 81:716-724. https://doi.org/10.1016/j.chemospher e.2010.07.061

Wickstead B, Gull K (2011) The evolution of the cytoskeleton. J Cell Biol 194:513-525. https://doi.org/10.1083/jcb.201102065

Xie L, Xie P, Guo L et al (2005) Organ distribution and bioaccumulation of microcystins in freshwater fish at different trophic levels from the eutrophic Lake Chaohu, China. Environ Toxicol 20:293300. https://doi.org/10.1002/tox.20120

Zegura B (2016) An overview of the mechanisms of microcystin-LR genotoxicity and potential carcinogenicity. Mini-Rev Med Chem 16:1042-1062. https://doi.org/10.2174/13895575166661603081 41549

Zegura B, Sedmak B, Filipic M (2003) Microcystin-LR induces oxidative DNA damage in human hepatoma cell line HepG2. Toxicon 41:41-48. https://doi.org/10.1016/S0041-0101(02)00207-6
Zegura B, Volcic M, Lah TT, Filipic M (2008) Different sensitivities of human colon adenocarcinoma ( $\mathrm{CaCo}-2)$, astrocytoma (IPDDCA2) and lymphoblastoid (NCNC) cell lines to microcystin-LR induced reactive oxygen species and DNA damage. Toxicon 52:518-525. https://doi.org/10.1016/j.toxicon.2008.06.026

Zegura B, Gajski G, Straser A et al (2011) Microcystin-LR induced DNA damage in human peripheral blood lymphocytes. Mutat Res Toxicol Environ Mutagen 726:116-122. https://doi.org/10.1016/j. mrgentox.2011.10.002

Zelko IN, Mariani TJ, Folz RJ (2002) Superoxide dismutase multigene family: a comparison of the CuZn-SOD (SOD1), MnSOD (SOD2), and EC-SOD (SOD3) gene structures, evolution, and expression. Free Radic Biol Med 33:337-349. https://doi. org/10.1016/S0891-5849(02)00905-X

Zhao Y, Xie P, Fan H (2012) Genomic profiling of microRNAs and proteomics reveals an early molecular alteration associated with tumorigenesis induced by MC-LR in mice. Environ Sci Technol 46:34-41. https://doi.org/10.1021/es201514h

Zhao S, Li G, Chen J (2015) A proteomic analysis of prenatal transfer of microcystin-LR induced neurotoxicity in rat offspring. J Proteomics 114:197-213. https://doi.org/10.1016/j.jprot.2014.11.015

Zhao S, Xie P, Chen J et al (2016) A proteomic study on liver impairment in rat pups induced by maternal microcystin-LR exposure. Environ Pollut 212:197-207. https://doi.org/10.1016/j.envpo 1.2015.12.055

Zhou M, Tu W, Xu J (2015) Mechanisms of microcystin-LR-induced cytoskeletal disruption in animal cells. Toxicon 101:92-100. https ://doi.org/10.1016/j.toxicon.2015.05.005

Publisher's Note Springer Nature remains neutral with regard to jurisdictional claims in published maps and institutional affiliations. 